\title{
Is fasting a necessary preparation for abdominal ultrasound? Tariq Sinan ${ }^{1}$, Hans Leven ${ }^{2}$ and Mehraj Sheikh*1
}

Address: ${ }^{1}$ Department of Radiology Faculty of Medicine, Kuwait University, Kuwait and ${ }^{2}$ Department of Radiology, Sultan Qaboos University Hospital, Muscat, Oman

Email: Tariq Sinan -drtariq999@yahoo.com; Hans Leven - hansol@squ.edu.om; Mehraj Sheikh* - mehraj@hsc.kuniv.edu.kw

* Corresponding author

Published: 22 July 2003

BMC Medical Imaging 2003, 3:I

This article is available from: http://www.biomedcentral.com/I47I-2342/3/I
Received: 16 March 2003

Accepted: 22 July 2003 \footnotetext{
media for any purpose, provided this notice is preserved along with the article's original URL.

(C) 2003 Sinan et al; licensee BioMed Central Ltd. This is an Open Access article: verbatim copying and redistribution of this article are permitted in all
}

\begin{abstract}
Objective: To study the effect of fasting on the technical success of abdominal ultrasound examination.

Methods: In a randomized, prospective study, I 50 patients for abdominal ultrasound were divided into two groups of 75 patients each with instructions to fast for six hours or have normal breakfast respectively.
\end{abstract}

Result: The technical success of the abdominal ultrasound performed by radiologists blinded to the instruction did not differ significantly between the groups.

Conclusion: It appears that routine fasting before abdominal ultrasound is not necessary.

\section{Introduction}

Optimum conditions for the ultrasonic examination of the abdominal organs require a fluid-filled gall bladder and as little gas in the gastrointestinal tract as possible [1]. In addition the thin patient is easier to examine than the fat patient since higher-frequency transducers can be used which improve resolution. Most ultrasound (US) departments instruct their patients to come fasting for such examinations, sometimes for as long as 12 hours, assuming that the gall bladder would otherwise be contracted and difficult to assess and that fasting patients will have less gas in the duodenum \& colon. Vogel et al [1] have reported favourable results with a two-day low calorie diet, laxatives and fasting. Other groups have found gas reducing agents to be of no value [2]. Some investigations have found a barium meal or upper gastrointestinal series does not interfere with abdominal US although the US images, especially of the liver and gall bladder $[3,4]$ were poor after barium enema.

We have been unable to find any report on the technical outcome of abdominal US in non-fasting patients. Fasting may be inconvenient and even dangerous for some patients, particularly diabetics, and those having to travel a long way to the US department. The effect of fasting is compounded by waiting and some patients are psychologically affected by low blood sugar. Also, if a urinary bladder US examination is to follow an abdominal examination, it will take longer for the urinary bladder to fill in a fasting and poorly hydrated patient. Non-fasting may also be used as an excuse to refuse an immediate US examination, which would often be more convenient for the patient. Most importantly, patient with critical hepatic \& pancreatic lesions may not receive appropriate treatment early enough. 
We have undertaken a prospective study to establish whether there is a real need for fasting before US examinations of the abdomen.

\section{Materials and Methods}

One hundred and fifty outpatients of Arabic and Indian origin, referred for abdominal US, aged 10-84 were randomly assigned for the study. Randomization was done by putting 75 notes with the test "Fasting" and 75 with the test "Non fasting" in 150 unmarked, identical envelopes. These were sealed and mixed thoroughly. They were then handed over to the department's reception clerk with instruction to open one envelope for each patient referred for abdominal US. The patient was given an instruction sheet for "fasting" or "non-fasting" as written on the note. The note was then returned to the envelope and a sequential number (1 150) was written on the envelope and on the request form. The envelopes were stored in the reception until the study was completed, when the radiologists' record of each examination was decoded against the numbered envelopes.

Seventy five patients received instructions to fast for six hours before the US examination which took place between 8 and 11 a.m. The remaining 75 patients were told to have their usual breakfast in the morning or had one already before the US examination. Five experienced radiologists performed abdominal US, using the same US machine (Toshiba SSA 270) with phased array and convex probes of $3.75 \mathrm{MHz}$ frequency. The radiologists were completely blinded to the patients' instructions and did not ask any questions about fasting status. Examinations were scored on a scale of 1-3 given for excellent (3), acceptable (2) and unacceptable (1) visualization of the gall bladder, common bile duct, pancreas, aorta and surrounding retroperitoneal space (with a transabdominal approach), kidneys and spleen (with retroperitoneal approach). "Excellent" scan quality denoted images which clearly define the anatomy of the organs and which we would have chosen for publication in an article or a textbook. "Acceptable" was defined as clinically adequate with no need to repeat the study and "Unacceptable" was used when the study was considered a technical failure and the patient had to be recalled for a repeat examination. For statistical analysis the scans that were "excellent" and the "acceptable" were grouped together and analysis was done using the Fisher exact test. The amount of gastrointestinal gas was defined depending on the visibility of the pancreas. If major part of the pancreas was visible without any intervening gas or was invisible due to intervening bowel gas, the bowel gas was described as "minimal" or "excessive" respectively. Any other category was considered "intermediate". The physiologic status vis fasting/ non-fasting was not disclosed to the examiner. In all patients where the pancreas \& retroperitoneum were obscured by bowel contents, a water load of $500 \mathrm{ml}$ of water was administered \& the area re-examined at the same sitting.

\section{Results}

Forty six of the randomized 150 patients had to be excluded from the study, 6 because of uncertainly about the instructions received and 40 because of failure to attend or because the corresponding envelopes were lost from the reception. One hundred and four patients remained for follow up. Two of the non-fasting patients had cholecystectomies. The quality of the US scans for the different organs is shown in Table 1. There was little difference between fasting and non-fasting patients. Seventeen times a study was determined unacceptable (in 12 patients). Nine of these studied were in a fasting and eight in a non-fasting object. Sixteen unacceptable studies were noted in patients with a marked or excessive amount of gas in the gastrointestinal tract while only 7 were in patients with marked or excessive abdominal obesity, and all of these 7 overlapped with the 16 "gassy" cases. Statistical analysis revealed that there was no significant difference in the amount of gas between the fasting and nonfasting patients but more of the fasting group were obese. Twelve (12\%) patients had unacceptable studies of whom 6 were fasting and 6 non-fasting patients.

\section{Discussion}

Our patients of Arabic and Indian origin usually have a light breakfast - typically coffee or tea, bread, cheese and dates. It is not surprising that such a meal, taken some two to four hours before an abdominal US examination does not have a significant impact on the technical success. Admittedly the single unacceptable score for the gall bladder was in a patient with a contracted gall bladder who unfortunately failed to return for a repeat study. This patient was not fasting at initial presentation. The gall bladders in the other 49 patients in the non-fasting group were easily assessed. The patterns of gall bladder emptying have been found to vary considerably even in the same healthy individual [5] but there are no investigations of the optimal gall bladder size for US scanning and how long it takes to refill after maximal contraction. In only 1 patient was the visualization of the CBD unacceptable and this was in a post cholecystectomized patient with worst scores for CBD, pancreas and aorta/ retroperitoneal space and excessive gas in the GI tract. This patient was also not fasting. Of a total of 8 patients with unacceptable US visibility of the aorta and retroperitoneal space ( 4 fasting and 4 non-fasting) all had marked or excessive GI gas, while only 4 had excessive abdominal obesity and these 4 overlapped with the 8 with excessive GI gas.

Obesity was not correlated with impaired pancreatic visibility at all, while there was a strong correlation between 
Table I: Visualization of the gallbladder, common bile duct, pancreas, aorta/retroperitoneal space, kidneys and spleen in fasting and non-fasting patients

\begin{tabular}{|c|c|c|c|c|c|}
\hline & Excellent & Acceptable & Unacceptable & $\mathbf{N}$ & Total \\
\hline \multicolumn{6}{|c|}{ GALLBLADDER* } \\
\hline Fasting & $44(88)$ & $6(12)$ & 0 & 50 & \\
\hline Non-fasting & $44(84)$ & $7(14)$ & $\mathrm{I}(2)$ & 52 & 102 \\
\hline \multicolumn{6}{|l|}{ CBD } \\
\hline Fasting & $44(85)$ & $8(15)$ & 0 & 52 & \\
\hline Non-fasting & $42(89$ & $9(17)$ & $I(2)$ & 52 & 104 \\
\hline \multicolumn{6}{|c|}{ PANCREAS } \\
\hline Fasting & $34(65)$ & $13(25)$ & $5(10)$ & 52 & \\
\hline Non-fasting & $29(56)$ & $21(40)$ & $2(4)$ & 52 & 104 \\
\hline \multicolumn{6}{|c|}{ AORTA/RETROPER.SPACE } \\
\hline Fasting & $21(40)$ & $27(52)$ & $4(8)$ & 52 & \\
\hline Non-fasting & $23(44)$ & $25(48)$ & $4(8)$ & 52 & 104 \\
\hline \multicolumn{6}{|l|}{ KIDNEYS } \\
\hline Fasting & $5 I(98)$ & 0 & $\mathrm{I}(2)$ & 52 & \\
\hline Non-fasting & $51(98)$ & 0 & $I(2)$ & 52 & 104 \\
\hline \multicolumn{6}{|l|}{ SPLEEN } \\
\hline Fasting & $48(92)$ & 0 & $4(8)$ & 52 & \\
\hline Non-fasting & $50(96)$ & 0 & $2(4)$ & 52 & 104 \\
\hline
\end{tabular}

(percentages in brackets) [ $* 2$ fasting patients had cholecystectomies] ["Excellent" and "acceptable" groups combined for $2 \times 2$ table analysis versus the unacceptable group, using Fisher exact test : cutoff for significance 0.05]

worst scoring pancreas results and the amount of GI gas. The kidneys and spleen being extra-peritoneal organs allowing acoustic access which avoids the GI tract all had acceptable scans with a high percentage of excellent visibility. Of the 2 acceptable but not excellent kidney scores, 1 was in an excessively obese patient and out of 6 acceptable, but not excellent spleen scans, 2 were in excessively obese patients. As far as obesity is concerned the choice of frequency is a well known factor. We performed all our scans with $3.75 \mathrm{MHz}$ probes.

\section{Conclusion}

In conclusion our study has shown that a light breakfast is compatible with a satisfactory technical outcome of abdominal US scanning a few hours later. It illustrates the fact that visibility of abdominal organs did not differ between fasting and non-fasting patients. Obesity had little impact on the results. It appears from this study that fasting before abdominal ultrasound examination is not necessary.

\section{Competing Interests}

None declared

\section{Author's Contributions}

Author HL designed the study and provided overall supervision. Authors TS and MS drafted the manuscript, MS did the statistical analysis.

\section{Acknowledgement}

We are grateful to Delphine D'souza for providing secretarial assistance.

\section{References}

I. Vogel HJPH, Schipper J and Hermans J: Abdominal Ultrasonography : Improved Image Quality with the combined use of a diet and laxatives J Clin Ultrasound 1990, 18:627-30.

2. Heldwein W, Sommerlutte TH, Hasford J, Lehnert P, Littig G and Muller-Lissner S: Evaluation of the usefulness of Dimethicone and/or Senna Extract in improving the visualization of abdominal organs J Clin Ultrasound 1987, I 5:455-58.

3. Elam EA, Hunter TB, Hunt KR, Fajardo LL, Boren W and Gaines J: The lack of Sonographic Image Degradation after Barium Upper Gastrointestinal Examination AJR 1989, I 53:993-994.

4. Hunter TB, Elam EA, Hunt KR and Fajardo LL: Sonographic Image Degradation after Barium Enema Investigative Radiology 1993, 28:295-96.

5. Donald JJ, Fache JS, Bucklet AR and Burhenne HJ: Gallbladder contractility Variation in normal subjects AJR 199I, I57:753-56.

\section{Pre-publication history}

The pre-publication history for this paper can be accessed here:

http://www.biomedcentral.com/1471-2342/3/1/prepub 